\title{
Optimizing Performance of Non-Expert Users in Brain-Computer Interaction by Means of an Adaptive Performance Engine
}

\author{
André Ferreira $^{1}$, Athanasios Vourvopoulos ${ }^{1}$, and Sergi Bermúdez i Badia ${ }^{1}$ \\ ${ }^{1}$ Madeira-ITI, Universidade da Madeira (UMa), Funchal, Portugal \\ \{andre.ferreira, athanasios.vourvopoulos, sergi.bermudez \\ am-iti.org \\ 9000-390 Madeira, Portugal
}

\begin{abstract}
Brain-Computer Interfaces (BCIs) are become increasingly more available at reduced costs and are being incorporated into immersive virtual environments and video games for serious applications. Most research in BCIs focused on signal processing techniques and has neglected the interaction aspect of BCIs. This has created an imbalance between BCI classification performance and online control quality of the $\mathrm{BCI}$ interaction. This results in user fatigue and loss of interest over time. In the health domain, BCIs provide a new way to overcome motor-related disabilities, promoting functional and structural plasticity in the brain. In order to exploit the advantages of BCIs in neurorehabilitation we need to maximize not only the classification performance of such systems but also engagement and the sense of competence of the user. Therefore, we argue that the primary goal should not be for users to be trained to successfully use a BCI system but to adapt the BCI interaction to each user in order to maximize the level of control on their actions, whatever their performance level is. To achieve this, we developed the Adaptive Performance Engine (APE) and tested with data from 20 naïve BCI users. APE can provide user specific performance improvements up to approx. $20 \%$ and we compare it with previous methods. Finally, we contribute with an open motor-imagery datasets with 2400 trials from naïve users.
\end{abstract}

Keywords: Brain-Computer Interfaces · Adaptive Performance · Motor Imagery

\section{Introduction}

Brain-computer interfaces (BCIs) are systems which aim at providing users with alternative communication channels. BCIs detect changes in brain signals and translate them into control commands [1]. Such systems utilize well defined underlying relationships between users' mental state and corresponding electrophysiological signals. In non-invasive BCI's, the use of electroencephalography (EEG) is commonly used for measuring brain activity. Currently, the 3 main techniques for user interaction and control include: (a) Steady State Visual Evoked Potentials (SSVEP), (b) P300 BCI and (c) Motor-Imagery (MI) or Event Related Synchronization/Desynchronization BCI. The use of these techniques in health are divided into 
two groups: (1) assistive and (2) restorative [2]. An important distinction between the two strategies lies into the fact that assistive BCIs are based on "replacing" the damaged motor mechanisms, and restorative on "improving" existing motor function. Assistive BCI's can provide humans with motor impairments like tetraplegia, an alternative channel for communication or control by bypassing the affected corticospinal pathways. Examples include the control of functional electrical stimulation (FES) [3], orthotic devices [4], EEG wheelchair control [5], or BCI spelling devices [6]. On the other hand, restorative BCIs, target at mobilizing plastic changes of the brain in order to achieve reorganization of motor networks and enhance motor recovery [7]. MI training based on visuo-motor imagination BCI is the most common type of BCI paradigm for motor function restoration. Results from previous studies have proven mental practice of action to be useful in MI-BCI [8], and have shown beneficial effects of MI practice during stroke recovery [9]. Overall, in neurorehabilitation, there is increasing evidence that technology-mediated therapy, like robotic and virtual reality based training [10], affects positively motor outcomes compared to standard rehabilitation techniques [11], [12]. So far, the combination of BCIs and virtual environments has gained popularity, and has been proven useful to train functional upper limb pointing movements [13], [14], although the use in clinical environment is limited [15] and hardly used outside laboratory environments [16]. This is mainly due to the fact that current BCI systems lack reliability and good performance in comparison with other types of interfaces [17]. As a result, there is no solid evidence on how BCI training needs to be designed and how improvements transfer to real life [18].

Additionally, within the last few years, the launch of low-cost EEG devices increased the user exposure and consequently the amount of BCI studies [19]. This rise in popularity led to the incorporation of BCIs as an alternative input to games, with early adoption by casual gamers. This has implications in terms of accessibility, level of control and BCI illiteracy [20]. Unfortunately, BCI training still requires long training periods resulting in user fatigue and low performance. This led Human-Computer Interaction researchers to work towards novel approaches to increase the communication bandwidth and quality of the BCI loop [21].

A comparative analysis on pure MI-BCI showed varying setups, algorithms and results [22]. Some studies report very different success rates using very similar approaches. Maximum performance scores of $89 \%$ were found on a bipolar montage (central electrodes over $\mathrm{C} 3, \mathrm{Cz}, \mathrm{C} 4$ ) classified through a Bayes quadratic based on data from one healthy subject [23]. The lowest performance reached $61 \%$ with the same montage but using Linear Discriminant Analysis (LDA) tested with data from 2 healthy subjects [24]. Overall, all studies had very small training datasets with 2 users on average. In addition, most users had been previously trained on BCI use. Consequently, the risk of overfitting is very high, what results in poor predictive performance for the general user when it comes to actual online control. This imbalance between theoretical training performance and actual quality of the online control experienced by the general non-expert user suggests a shift in the interaction paradigm. Current MI-BCI interaction relies on time-constrained binary decisions - such as left vs. right arm motor imagery - and users undergo long, tiresome and complex periods of training so that EEG classification algorithms can reach acceptable performance rates. Here we propose to reverse the problem at hand and make MI-BCI interaction adaptive to the user, so that we can guarantee a satisfactory performance rates by 
softening decisions - making them probabilistic and non-time-constrained - depending on our confidence on the user's EEG data. That is, we argue that the primary goal should not be for users to be trained to use a BCI system but to adapt the BCI interaction to each user in order to maximize the level of control on their actions. This will allow non-expert users and low-performing users to be able to increase their control, acceptance and motivation towards MI-BCI systems. To address the above limitations and improve MI-BCI based paradigms, with specific focus on motor rehabilitation, we have developed a MI-BCI Adaptive Performance Engine consisting on a Probabilistic Finite State Machine approach to increase sense of control as opposed to EEG classifier performance. Our system has been tested on 20 naïve healthy subjects, allowing for up to approx. $20 \%$ increase on performance when compared to standard EEG classification performance.

\section{Methodology}

\subsection{Data Acquisition and Training Datasets}

The BCI set up comprised 8 active electrodes equipped with a low-noise g.MOBIlab biosignal amplifier (gtec, Graz, Austria) and a 16-bit A/D converter (256 $\mathrm{Hz}$ ). The spatial distribution of the electrodes followed the 10-20 system configuration [25] with the following electrodes over the sensory-motor areas: FC3, FC4, C3, C4, C5, C6, CP3, CP4. For all user data, a common spatial patterns filter was used for feature extraction, and LDA for the classification of MI from EEG data. The visual stimulation was based on the Graz-BCI paradigm [26] with a standard bars-andarrows feedback on a binary (left vs. right) MI paradigm.

Experimental data consisted of a set of 20 EEG datasets consisting of 120 trials each, acquired from 20 healthy users $(28 \pm 4)$ performing standard MI training. Participants had no previous known neurological disorder and no previous experience in BCIs. Participants gave informed consent. Data from the MI datasets was processed in Matlab (MathWorks Inc., Massachusetts, US).

\subsection{BCI - Adaptive Performance Engine}

The BCI Adaptive Performance Engine (BCI-APE) is composed by 2 main components: (a) a Bayesian Inference Layer (BIL), simpler and more efficient as compared to other supervised learning techniques such as artificial neural networks, in order to formulate the input into a model, where we translate the continuous BCI classification data into probability. As for decision making, we made use of a (b) Finite State Machine (FSM) because of its efficiency and non-linear properties.

\subsubsection{Bayesian Inference Layer}

BIL works on top of the LDA EEG classifier, and is used to compute the likelihood of the classifier output for each MI class (left vs. right). This is done by modeling the data belonging to each class as a Gaussian distribution, where $\mu$ and $\sigma$ indicate 
their mean and standard deviation values $\left(\operatorname{MI}_{i}(\mu, \sigma), i=[\right.$ left,right $\left.]\right)$. From it, we then compute the Likelihood of a specific LDA output belonging to each MI class with:

$P(i \mid L D A$ output $)=\frac{M I_{i}\left(\text { LDA output }, \mu_{i}, \sigma_{i}\right) * P_{i}}{\sum_{j} M I_{j}\left(\text { LDA output }, \mu_{j}, \sigma_{j}\right)}$

Where $P_{i}$ indicates the prior probability of action $i(0.5$ for left vs. right MI). $\mu$ and $\sigma$ are updated at each iteration, taking into account all previous history of the user for the given $i$ MI action. LDA output indicates the output value of the LDA classifier.

\subsubsection{Finite State Machine}

Following the BIL, the likelihood of each MI classification forwarded into a FSM. The role of the FSM is to transform binary MI classifications - such as left vs. right into evidence-based states $\left(\mathrm{S}_{\mathrm{i}}\right)$. Is composed of 7 states, a neutral $\left(\mathrm{S}_{0}\right)$ and three for each MI class $\left(\mathrm{S}_{1 /-1}, \mathrm{~S}_{2 /-2}, \mathrm{~S}_{3 /-3}\right)$. Each state has a transition threshold associated with it $\left(\mathrm{w}_{1}, \mathrm{w}_{2}, \mathrm{w}_{3}\right)$, and can only transition to one of the nearest neighbors or stay in the same state (see Fig.1). As input, the FSM uses the difference of the posterior probabilities of left and right MI from eq. 1 and each state represents not only the class (negative and positive states represent left and right MI respectively), but also the confidence level associated to them (being $\mathrm{S}_{3 /-3}$ the most certain states).

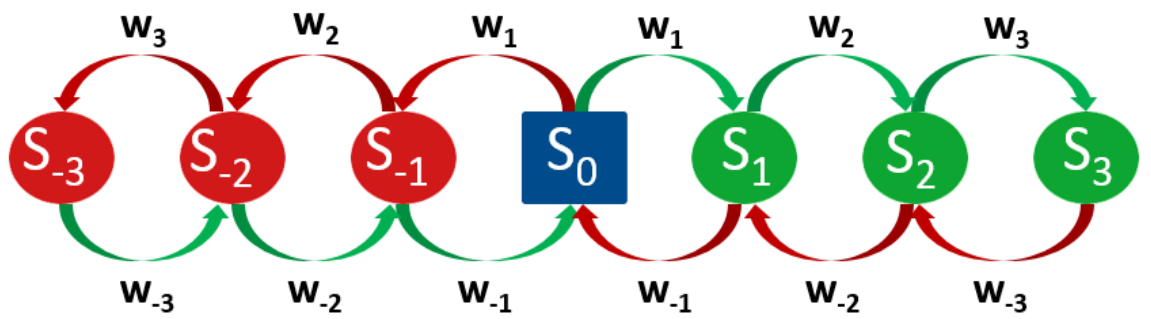

Fig. 1. State machine structure. $S_{0}$ represents the neutral state (indecision). The level of confidence of $\mathrm{S}_{-3,3}>\mathrm{S}_{-2,2}>\mathrm{S}_{-1,1}$, and $\mathrm{W}_{-3,-2,-1,1,2,3}$ are the state transition thresholds.

\section{Results}

\subsection{Can performance be improved by means of the BCI-APE approach?}

In order to answer this, we used a dataset with MI training sessions of 15 naïve users to explore the parameter space of the aforementioned state machine thresholds $\left(\mathrm{W}_{\mathrm{i}}\right)$ from 0 up to 0.3 on a 0.05 step, what resulted in 117649 FSM parameter combinations. For each combination we quantified the percentage of indecisions $\left(\mathrm{S}_{0}\right)$ and the correctness of decisions based on the remaining states. Results show that the FSM approach can increase performance over the original LDA classification (up to ap- 
prox. 20\%) at the expense of an increased amount of indecisions (Fig.2). That is, less decisions are taken but with higher confidence.

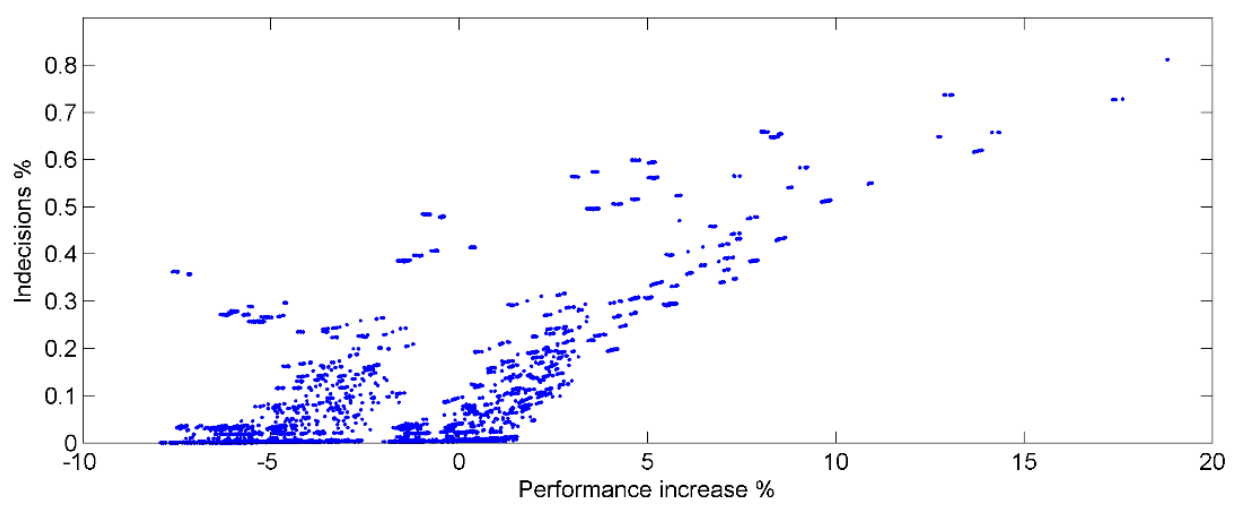

Fig. 2. Performance increase vs. indecisions percentage for the 117649 FSM parameter combinations on a MI dataset of 15 naïve users.

\subsection{What are the tradeoffs of an increased MI-BCI performance?}

Out of the above 117649 parameter combinations, we selected those combinations that provide the greater performance improvements with the minim number of indecisions. From these data we observe that exists a set of FSM parameters that can provide us a continuum from $0 \%$ performance increase and $0 \%$ indecisions - equivalent to standard LDA performance - to $20 \%$ performance increase and $80 \%$ indecisions (see Fig 3). As consequence, this means that we can devise an algorithm to adjust the FSM to tradeoff between decision time - or number of indecisions - and confidence on decisions.

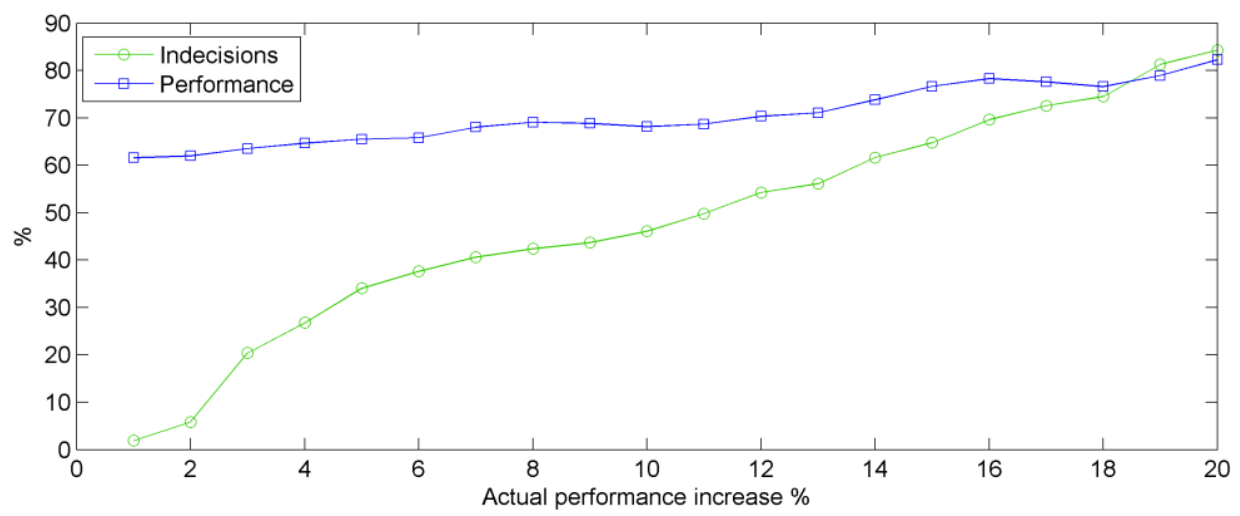

Fig. 3. Performance increase achieved with the FSM vs. indecisions. 


\subsection{Can APE adjust performance in real time?}

Cross-referencing the best performing FSM thresholds values $\left(\mathrm{W}_{\mathrm{i}}\right)$ with their resulting performance increase allows us to identify their relationship as illustrated in Fig.4(a). This is a crucial step, due to only a few FSM weight combinations actually resulting in increased performance. It was also found that $\mathrm{W}_{-1,-2,-3}$ should be kept 0 for maximal performance. That is, thresholds should be applied to transition from indecision to any decision state, but not to transition from any state to indecision. Further, it was also found that $\mathrm{W}_{3}$ should be kept always constant at the highest value $(0.3)$ and that $\mathrm{W}_{1}$ increases with the overall performance. Interestingly, the evolution of $\mathrm{W}_{2}$ with achieved performance shows that for low $\mathrm{W}_{1}$ values - easy to transition from indecision to the lowest confidence level of decision $-\mathrm{W}_{2}$ should be kept high whereas for high $\mathrm{W}_{1}$ values - difficult to transition from indecision to the lowest confidence level of decision $-\mathrm{W}_{2}$ should be low to facilitate the transition from low to mid confidence. We used a $3^{\text {rd }}$ degree polynomial function to model how $\mathrm{W}_{1}$ and $\mathrm{W}_{2}$ change depending on the achieved performance increases (x) [Fig.4(a)] (eq. 2):

$W_{1}=114.42 * x^{3}-36.517 * x^{2}+4.7014 * x-0.058208$
$W_{2}=87.662 * x^{3}-32.613 * x^{2}+2.4013 * x+0.16366$

(a)

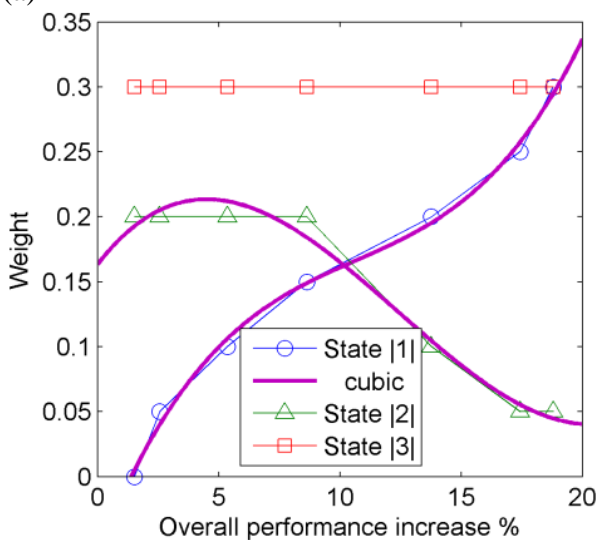

(b)

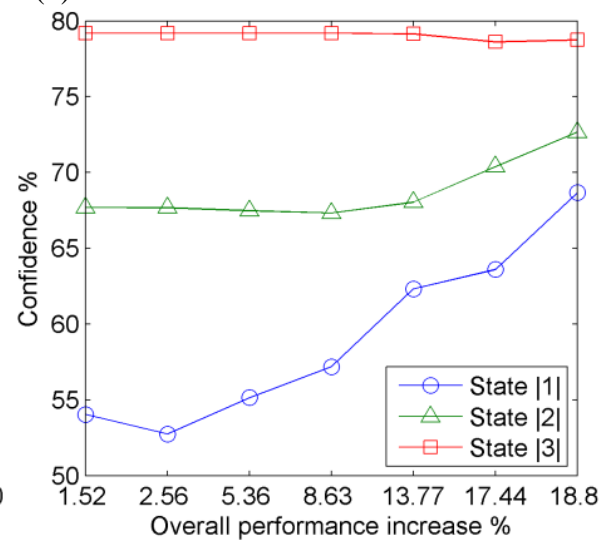

Fig. 4. (a) FSM threshold weight values vs. performance increase. (b) State confidence levels vs. performance increase.

Decisions taken at each state of the FSM have an associated performance level. That is, a MI detected based on $\mathrm{S}_{3}$ should be more certain than in $\mathrm{S}_{1}$. Fig.4(b) illustrates the confidence level associated with each State $\left(S_{3}>S_{2}>S_{1}\right)$, and how these change based on the FSM performance increase. In average, the confidence of $\mathrm{S}_{3}, \mathrm{~S}_{2}$, and $S_{1}$ is $79.04 \% \pm 0.25 \%, 68.76 \% \pm 2.02 \%$ and $59.1 \% \pm 5.88 \%$ respectively. 


\subsection{Evaluation of the complete BCI-APE system}

From the training data from $15 \mathrm{BCI}$ naïve users we obtained the following results: Classification performance with standard LDA $58.70 \% \pm 7.84 \%$; Average improved performance of BCI-APE 70.46\% $\pm 6.90 \%$; Average maximum performance of BCIAPE $85.37 \% \pm 10.09 \%$; and indecisions of BCI-APE $48.25 \% \pm 24.62 \%$. Further, we implemented the complete BIL + FSM based on the above models of performance increase and we tested it against a dataset from 5 different $\mathrm{BCI}$ naïve users containing $5 \times 120$ MI trials. The previous results are confirmed with the test data: Classification performance with standard LDA $63.93 \% \pm 6.28 \%$; Average improved performance of BCI-APE $71.83 \% \pm 6.64 \%$; Average maximum performance of BCI-APE $88.37 \% \pm$ $6.49 \%$; and indecisions of BCI-APE $38.82 \% \pm 19.60 \%$.

\section{Conclusions}

BCI-APE was created from the need of ensuring satisfactory performances for non-expert and low-performing BCI users. BCI-APE provides a way to adapt performance accuracy on demand depending on the specific needs of users. By means of the presented model for online adjustment of the FSM transition weights (eq. 2), the accuracy of standard BCI classification algorithms such as LDA can be boosted up to $20 \%$ by clustering low confidence data as an indecision state $\mathrm{S}_{0}$. Thus, for a specific BCI task a minimum acceptable performance rate can be stipulated, and by means of BCIAPE the performance rate of each user can be adjusted ( $0-20 \%$ performance increase) to guarantee that all users can have a satisfactory experience. Thus, better performing users will have less indecisions and response times will be faster than those lowperforming BCI users. However, overall success rate will be comparable. Further, the confidence of a specific MI action detections is stratified in states $\left(S_{3}>S_{2}>S_{1}\right)$ enabling designers of BCI tasks - such as neurofeedback, restorative, mental training or games - to decide what is best to do when confidence on a detection is small. Thus, effectively empowering them with tools to enhance usability and improve the experience of BCI users.

\section{Discussion and Future Work}

Solid and systematic improvements are seen when comparing the performance achieved by APE with LDA. Existing MI-BCI classification approaches are very dissimilar in setup, algorithms, user experience, datasets, etc. This makes it very difficult to assess if differences in performance arise from training, users, algorithms or setup. Thus, it results impossible to establish what the most appropriate MI-BCI classification approach is best. Nevertheless, when comparing BCI-APE to previous approaches - working on top of an LDA classifier and with naïve users - we observe a comparable performance with the best BCI classification algorithms (See Table 1). Further, we would expect even higher performances and lower indecisions if APE would be combined with more sophisticated and better performing classifiers than LDA. 
The obtained results show interesting findings in several dimensions related with the use of adaptive performance for MI-BCI. Firstly, this system provides a real time performance (or task difficulty) adaptation. This is important in order to balance the difficulty in terms of user control and contribute positively on the interaction level by modulating user frustration/engagement related to a certain task. If used within a game, it can provide an enjoyable experience, but when used on a rehabilitation scenario, it is of a paramount importance. Many times, in rehabilitation exercises are not performed with the correct frequency or intensity because of lack of motivation and engagement of the patient. The BCI-APE approach can be used to tackle this issue, making the patient more prone to complete the rehabilitation task at hand.

Furthermore, we identified that there is an important trade-off for this performance increase, and it comes in the form of less decisions for the same time window. Thus, depending on the response time and accuracy required, with the help of the APE model we can adjust the performance levels in real-time. Finally, given the lack of availability of large MI datasets containing naïve subjects, we submitted our dataset (20 users x 120 trials) on PhysioNet ${ }^{1}$ and made available under the Public Domain for dissemination and ex-change within the community.

Table 1. Classifier performance comparison, including APE. Adapted from Lotte et al.[22]

\begin{tabular}{|c|c|c|c|c|}
\hline Protocol & Classification & Accuracy & electrodes / channels & $\begin{array}{l}\text { \# of subjects, trials/subject, } \\
\text { training set, test set }\end{array}$ \\
\hline \multirow{14}{*}{$\begin{array}{l}\text { on different } \\
\text { EEG data }\end{array}$} & Gaussian SVM & $86 \%$ & \multirow{2}{*}{$2(\mathrm{C} 3, \mathrm{C} 4)$ at $128 \mathrm{~Hz}$} & \multirow{2}{*}{$\begin{array}{l}\text { 2, } 1000(500 / \text { side }), 400(200 \\
\text { random/side, remaining } 600\end{array}$} \\
\hline & LDA & $61 \%$ & & \\
\hline & LDA & $83.6 \%$ & \multirow{4}{*}{$\begin{array}{c}6 \text {--> } 3 \text { bipolar EEG } \\
\text { channels }\end{array}$} & \multirow{4}{*}{$\begin{array}{c}5 \text { familiar with the Graz-BCl, } \\
360,240 \text {, remaining without } \\
\text { artifacts }\end{array}$} \\
\hline & Boosting wth MLP's & $76.4 \%$ & & \\
\hline & LDA & $80.6 \%$ & & \\
\hline & Boosting wth MLP's & $80.4 \%$ & & \\
\hline & HMM & $81.4 \% \pm 12.8 \%$ & \multirow{2}{*}{$\begin{array}{c}4-> \\
\text { channels }\end{array}$} & \multirow{2}{*}{$\begin{array}{l}4 \text { ( } 3 \text { familiar and } 1 \text { naive), } \\
880, \text { some sessions where } \\
\text { used to train other sessions }\end{array}$} \\
\hline & LDA & $72.4 \% \pm 8.6 \%$ & & \\
\hline & MLP & $85.97 \%$ & \multirow{2}{*}{$\begin{aligned} 4--> & 2 \text { bipolar EEG } \\
& \text { channels }\end{aligned}$} & \multirow{2}{*}{$3,667 \pm 107,60 \%, 20 \%$} \\
\hline & FIR NN & $87.4 \%$ & & \\
\hline & HMM & $75.7 \%$ & \multirow{2}{*}{$\begin{array}{c}2 \text { bipolar EEG channels } \\
(\mathrm{C} 3, \mathrm{C} 4)\end{array}$} & \multirow{2}{*}{-,160 (80/side), -, - } \\
\hline & HMM + SVM & $78.15 \%$ & & \\
\hline & LDA & $65 \% \pm 3.3 \%$ & $\begin{array}{c}8 \text { electrodes (FC3, FC4, } \\
\mathrm{C} 3, \mathrm{C} 4, \mathrm{C} 5, \mathrm{C} 6, \mathrm{CP} 3, \mathrm{CP} 4)\end{array}$ & $12,120,-,-$ \\
\hline & $\mathrm{LDA}+\mathrm{BCl}-\mathrm{APE}$ & $\begin{array}{c}\text { up to } 87.49 \pm \\
7.13 \%\end{array}$ & $\begin{array}{c}8 \text { electrodes (FC3, FC4, } \\
\text { C3, C4, C5, C6, CP3, CP4) }\end{array}$ & $\begin{array}{c}20,120,15 \text { subjects, } 5 \\
\text { subjects }\end{array}$ \\
\hline \multirow{9}{*}{$\begin{array}{c}\text { on } \mathrm{BCl} \\
\text { competition } \\
2003 \text { data set } \\
\text { III }\end{array}$} & $\begin{array}{l}\text { Bayes quadratic } \\
\text { integrated over time }\end{array}$ & $89.3 \%$ & $\begin{array}{c}3 \text { bipolar EEG channels } \\
(\mathrm{C} 3, \mathrm{Cz}, \mathrm{C} 4)\end{array}$ & $1,140,-,-$ \\
\hline & BGN & $83.57 \%$ & \multirow{3}{*}{$\begin{array}{c}3 \text { bipolar EEG channels } \\
(\mathrm{C} 3, \mathrm{Cz}, \mathrm{C} 4)\end{array}$} & \multirow{3}{*}{$1,140,-,-$} \\
\hline & MLP & $84.29 \%$ & & \\
\hline & Bayes quadratic & $82.86 \%$ & & \\
\hline & HMM & up to $77.5 \%$ & $\begin{array}{c}3 \text { bipolar EEG channels } \\
(\mathrm{C} 3, \mathrm{Cz}, \mathrm{C} 4)\end{array}$ & $1,140,100,40$ \\
\hline & Gaussian classifier & $65.4 \%$ & \multirow{4}{*}{$\begin{array}{c}3 \text { bipolar EEG channels } \\
\text { (C3, Cz, C4) }\end{array}$} & \multirow{4}{*}{$1,140,100,40$} \\
\hline & LDA & $65.6 \%$ & & \\
\hline & Bayes quadratic & $63.4 \%$ & & \\
\hline & Mahalanobis distance & $63.1 \%$ & & \\
\hline
\end{tabular}

\footnotetext{
${ }^{1}$ http://physionet.org/
} 
As future work, new interaction paradigms need to be developed to embrace BCIAPE and to study its impact in users' perceived performance.

Acknowledgements: This work is supported by the European Commission through the RehabNet project - Neuroscience Based Interactive Systems for Motor Rehabilitation - EC (303891 RehabNet FP7-PEOPLE-2011-CIG), and by the Fundação para a Ciência e Tecnologia (Portuguese Foundation for Science and Technology) through SFRH/BD/97117/2013, and Projeto Estratégico - LA 9 - 2014-2015.

\section{References}

[1] J. R. Wolpaw, N. Birbaumer, D. J. McFarland, G. Pfurtscheller, and T. M. Vaughan, "Brain-computer interfaces for communication and control," Clin. Neurophysiol. Off. J. Int. Fed. Clin. Neurophysiol., vol. 113, no. 6, pp. 767-791, Jun. 2002.

[2] S. R. Soekadar, N. Birbaumer, and L. G. Cohen, "Brain-Computer Interfaces in the Rehabilitation of Stroke and Neurotrauma," in Systems Neuroscience and Rehabilitation, K. Kansaku and L. G. Cohen, Eds. Springer Japan, 2011, pp. 3-18.

[3] G. Pfurtscheller, G. R. Müller-Putz, J. Pfurtscheller, and R. Rupp, "EEG-Based Asynchronous BCI Controls Functional Electrical Stimulation in a Tetraplegic Patient," EURASIP J. Adv. Signal Process., vol. 2005, no. 19, p. 628453, Nov. 2005.

[4] G. Pfurtscheller, C. Guger, G. Müller, G. Krausz, and C. Neuper, "Brain oscillations control hand orthosis in a tetraplegic," Neurosci. Lett., vol. 292, no. 3, pp. 211-214, Oct. 2000.

[5] T. Carlson and J. del R Millan, "Brain-Controlled Wheelchairs: A Robotic Architecture," IEEE Robot. Autom. Mag., vol. 20, no. 1, pp. 65-73, Mar. 2013.

[6] N. Birbaumer, N. Ghanayim, T. Hinterberger, I. Iversen, B. Kotchoubey, A. Kübler, J. Perelmouter, E. Taub, and H. Flor, "A spelling device for the paralysed," Nature, vol. 398, no. 6725, pp. 297-298, Mar. 1999.

[7] B. H. Dobkin, "Brain-computer interface technology as a tool to augment plasticity and outcomes for neurological rehabilitation," J. Physiol., vol. 579, no. Pt 3, pp. 637-642, Mar. 2007.

[8] G. Prasad, P. Herman, D. Coyle, S. McDonough, and J. Crosbie, “Applying a braincomputer interface to support motor imagery practice in people with stroke for upper limb recovery: a feasibility study," J. NeuroEngineering Rehabil., vol. 7, no. 1, p. 60, Dec. 2010.

[9] F. Pichiorri, G. Morone, M. Petti, J. Toppi, I. Pisotta, M. Molinari, S. Paolucci, M. Inghilleri, L. Astolfi, F. Cincotti, and D. Mattia, "Brain-computer interface boosts motor imagery practice during stroke recovery," Ann. Neurol., Feb. 2015.

[10] M. S. Cameirao, S. B. i Badia, E. D. Oller, and P. F. Verschure, "Neurorehabilitation using the virtual reality based Rehabilitation Gaming System: methodology, design, psychometrics, usability and validation," J. NeuroEngineering Rehabil., vol. 7, p. 48, Sep. 2010.

[11] K. Laver, S. George, S. Thomas, J. E. Deutsch, and M. Crotty, "Cochrane review: virtual reality for stroke rehabilitation,” Eur. J. Phys. Rehabil. Med., vol. 48, no. 3, pp. 523-530, Sep. 2012. 
[12] G. Saposnik, M. Levin, and Outcome Research Canada (SORCan) Working Group, "Virtual reality in stroke rehabilitation: a meta-analysis and implications for clinicians," Stroke J. Cereb. Circ., vol. 42, no. 5, pp. 1380-1386, May 2011.

[13] F. Cincotti, F. Pichiorri, P. Aricò, F. Aloise, F. Leotta, F. de Vico Fallani, J. del R. Millán, M. Molinari, and D. Mattia, "EEG-based Brain-Computer Interface to support post-stroke motor rehabilitation of the upper limb," Conf. Proc. Annu. Int. Conf. IEEE Eng. Med. Biol. Soc. IEEE Eng. Med. Biol. Soc. Annu. Conf., vol. 2012, pp. 4112-4115, 2012.

[14] S. W. Tung, C. Guan, K. K. Ang, K. S. Phua, C. Wang, L. Zhao, W. P. Teo, and E. Chew, "Motor imagery BCI for upper limb stroke rehabilitation: An evaluation of the EEG recordings using coherence analysis," Conf. Proc. Annu. Int. Conf. IEEE Eng. Med. Biol. Soc. IEEE Eng. Med. Biol. Soc. Annu. Conf., vol. 2013, pp. 261-264, 2013.

[15] K. K. Ang and C. Guan, "Brain-Computer Interface in Stroke Rehabilitation," Journal of Computer Science and Engineering, Jun. 2013.

[16] F. Lotte, F. Larrue, and C. Mühl, "Flaws in current human training protocols for spontaneous Brain-Computer Interfaces: lessons learned from instructional design," Front. Hum. Neurosci., vol. 7, Sep. 2013.

[17] F. Lotte, "On the need for alternative feedback training approaches for BCI," presented at the Berlin Brain-Computer Interface Workshop, 2012.

[18] S. Silvoni, A. Ramos-Murguialday, M. Cavinato, C. Volpato, G. Cisotto, A. Turolla, F. Piccione, and N. Birbaumer, "Brain-Computer Interface in Stroke: A Review of Progress," Clin. EEG Neurosci., vol. 42, no. 4, pp. 245-252, Oct. 2011.

[19] D. Marshall, D. Coyle, S. Wilson, and M. Callaghan, "Games, Gameplay, and BCI: The State of the Art," IEEE Trans. Comput. Intell. AI Games, vol. 5, no. 2, pp. 82-99, Jun. 2013.

[20] B. Z. Allison and C. Neuper, "Could Anyone Use a BCI?," in Brain-Computer Interfaces, D. S. Tan and A. Nijholt, Eds. Springer London, 2010, pp. 35-54.

[21] D. Tan and A. Nijholt, "Brain-Computer Interfaces and Human-Computer Interaction," in Brain-Computer Interfaces, D. S. Tan and A. Nijholt, Eds. Springer London, 2010, pp. 319.

[22] F. Lotte, M. Congedo, A. Lécuyer, F. Lamarche, and B. Arnaldi, "A review of classification algorithms for EEG-based brain-computer interfaces," J. Neural Eng., vol. 4, no. 2, pp. R1-R13, Jun. 2007.

[23] S. Lemm, C. Schäfer, and G. Curio, "BCI Competition 2003--Data set III: probabilistic modeling of sensorimotor mu rhythms for classification of imaginary hand movements," IEEE Trans. Biomed. Eng., vol. 51, no. 6, pp. 1077-1080, Jun. 2004.

[24] G. N. Garcia, T. Ebrahimi, and J. Vesin, "Support vector EEG classification in the Fourier and time-frequency correlation domains," in First International IEEE EMBS Conference on Neural Engineering, 2003. Conference Proceedings, 2003, pp. 591-594.

[25] "Report of the committee on methods of clinical examination in electroencephalography: 1957," Electroencephalogr. Clin. Neurophysiol., vol. 10, no. 2, pp. 370-375, May 1958.

[26] G. Pfurtscheller, C. Neuper, G. R. Müller, B. Obermaier, G. Krausz, A. Schlögl, R. Scherer, B. Graimann, C. Keinrath, D. Skliris, M. Wörtz, G. Supp, and C. Schrank, "Graz-BCI: state of the art and clinical applications," IEEE Trans. Neural Syst. Rehabil. Eng. Publ. IEEE Eng. Med. Biol. Soc., vol. 11, no. 2, pp. 177-180, Jun. 2003. 